\title{
In vitro effects of aspartic acid, coenzyme Q10 and zinc on sperm function
}

\author{
Filippo Giacone, Rosita A. Condorelli, Sandro La Vignera, Aldo E. Calogero \\ Department of Clinical and Experimental Medicine, University of Catania, Catania, Italy
}

\section{Introduction}

D-aspartic acid (D-AA) is involved in the regulation of spermatogenesis by influencing hormonal biosynthesis and secretion, resulting in an increased sperm concentration and progressive motility. Coenzyme Q10 (CoQ10) correlates positively with sperm concentration and motility at high concentrations, and it plays an antioxidant role, improving sperm motility and fertilization rate in ICSI cycles. Finally, low concentrations of zinc ( $\mathrm{Zn})$ have been found in azoospermic patients, while higher concentrations improve sperm motility, concentration and viability. On these premises, the aim of this study was to evaluate the in vitro effects of these three compounds on spermatozoa

\section{Methods}

We enrolled:

- 10 patients with oligo-asthenoteratozoospermia (OAT)

- 5 normozoospermic subjects without infection/inflammation of the male accessory glands, varicocele or other andrological diseases

Spermatozoa were incubated for 3 hours with:

- BWW (group A),

- BWW+ethanol+PBS (group A1) (control groups)

- D-AA (500 mg/ml), CoQ10 (40 $\mathrm{mg} / \mathrm{ml}$ ) and $\mathrm{Zn}(10 \mathrm{~g} / \mathrm{ml}$ ) (group B)

After incubation, the following parameters were assessed: sperm progressive motility, non conventional sperm parameters (mitochondrial membrane potential, degree of viability and/or apoptosis, sperm DNA fragmentation, lipid peroxidation). Subsequently, sperm selection was performed by swim-up technique in all groups

\section{Results}

Effects on sperm progressive motility, number of motile spermatozoa recovered by swim-up and sperm lipid peroxidation in patients with OAT are reported in Figure 1.

The other sperm parameters examined did not change. No significant change was observed in normozoospermic subjects. Fig 1. Percentage of spermatozoa with progressive motility (A), Number of spermatozoa recovered by
swim-up (B) and percentage of spermatozoa with lipid peroxidation (C) in OAT patients

A

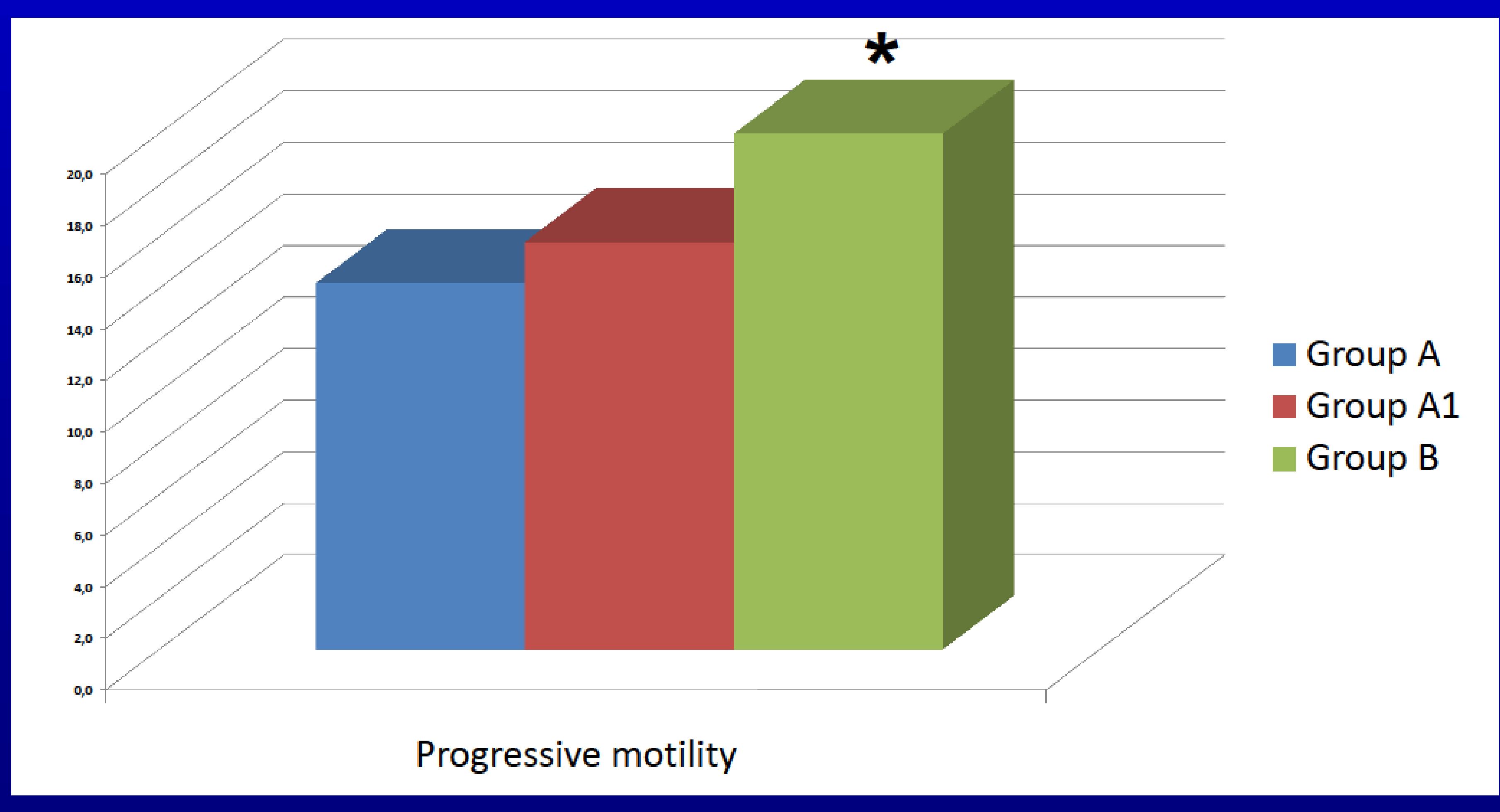

B

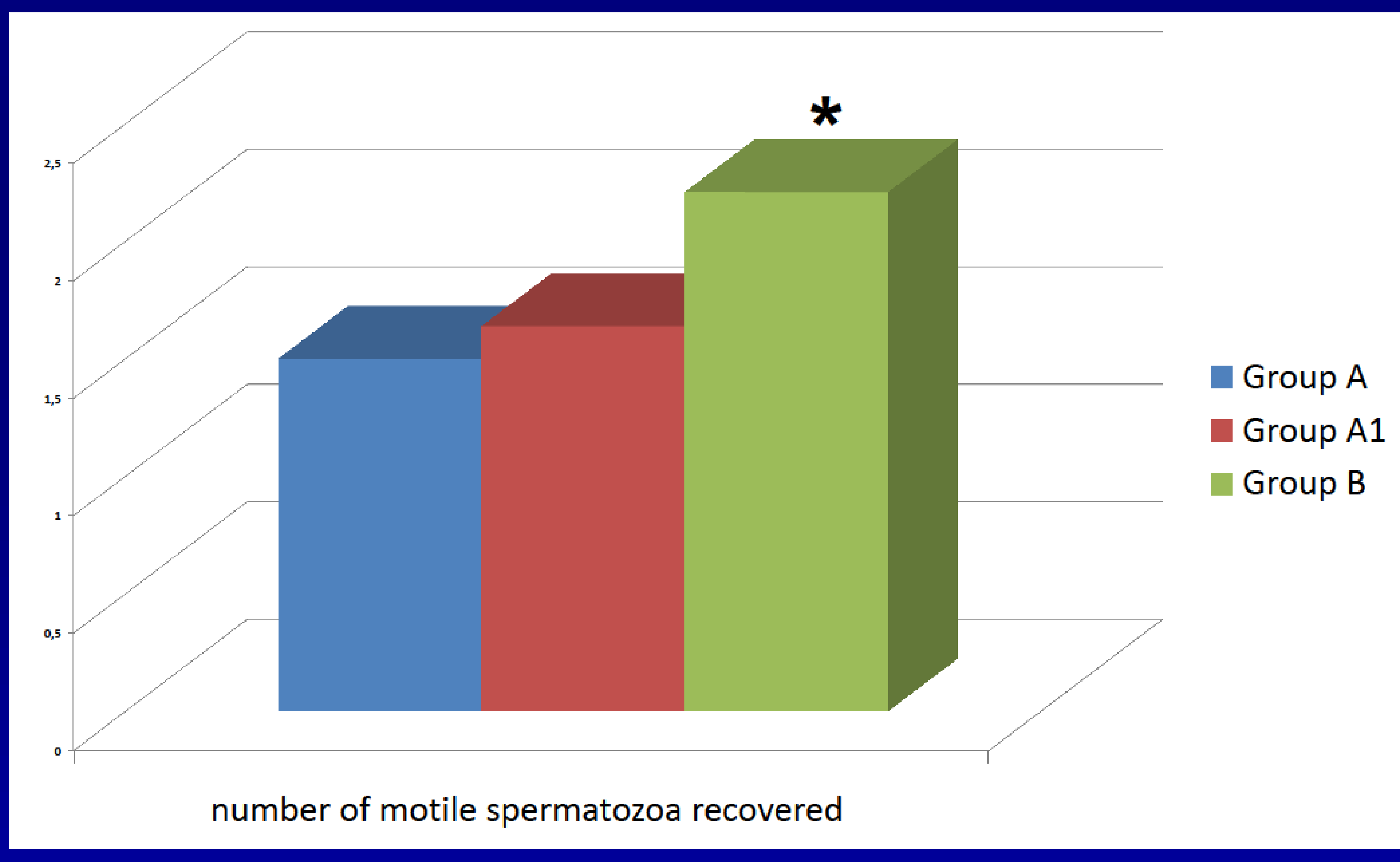

C

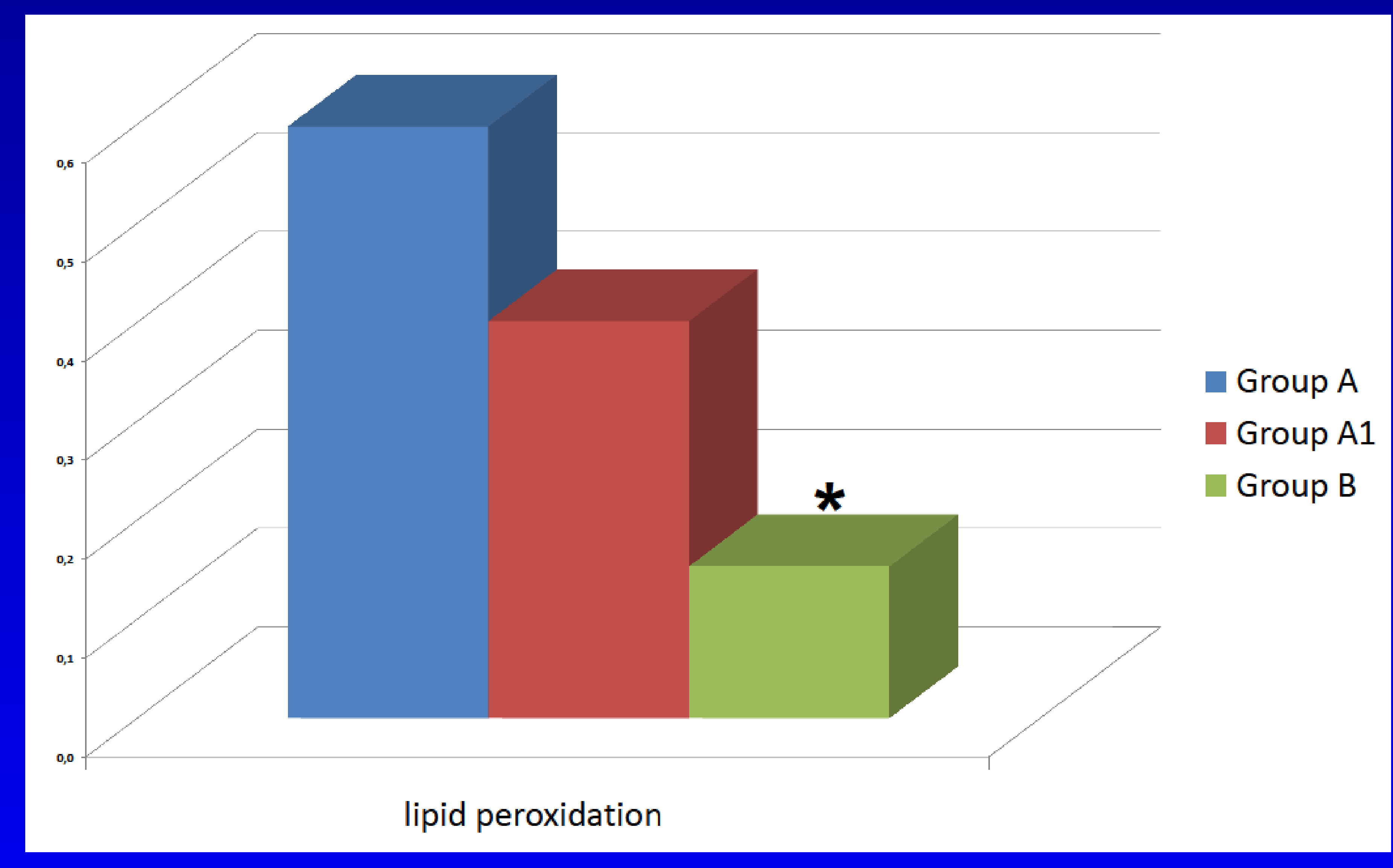

\section{Conclusions}

These results showed that $D-A A+C o Q 10+Z n$ act with antioxidant mechanism, ensuring a higher rate of motile spermatozoa recovered after swim-up, probably related to the improvement of progressive motility. These results suggest the use of this combination in both in vivo- and in vitro-assisted reproductive techniques. 